\title{
Revisión de estudios pre-clínicos de candidatos a vacuna contra la malaria causada por Plasmodium falciparum
}

\author{
Anny Jineth Camargo-Mancipe1, Diana Díaz-Arévalo², David Ricardo Salamanca- Jiménez', Laura \\ Esperanza Cuy-Chaparro', Diego Fernando Camargo-Mancipe ${ }^{1}$
}

\section{RESUMEN}

Introducción. La malaria es una enfermedad que causa aproximadamente 400.000 muertes al año, especialmente en niños menores de 5 años; la búsqueda de una vacuna eficaz y segura sigue siendo un reto para los investigadores, sin embargo, antes de iniciar los estudios de fase clínica, los ensayos preclínicos en modelo animal deben brindar resultados de seguridad e inmunogenicidad que lleven a respuestas eficaces de protección.

Objetivo. Revisar las principales características de la respuesta inmunológica y eficacia en estudios pre-clínicos de candidatos a vacuna contra la malaria por Plasmodium falciparum.

Métodos. Revisión descriptiva de los principales estudios preclínicos de candidatos a vacuna contra la malaria, basados en subunidades, parásitos atenuados y vacunas multi-estadio, multi-epitope, que se han realizado para evaluar inmunogenicidad y eficacia en modelo animal. Esta revisión se llevó a cabo a partir de la búsqueda de literatura en bases de datos electrónicas especializadas en investigación científica. Se encontraron 118 documentos, de los cuales se seleccionaron 91 y se excluyeron 17 por no cumplir con los criterios de inclusión, para un total de 74 referencias analizadas. Resultados. Muchos candidatos a vacuna contra la malaria causada por Plasmodium falciparum han reportado resultados prometedores contra cepas homologas, sin embargo, ante el reto con cepas heterólogas la eficacia disminuye, por otra parte, la respuesta inmune y protectiva duradera continúa siendo un objetivo clave, convirtiéndose en una prioridad.

Conclusiones. Los estudios preclínicos en modelo animal son necesarios antes de avanzar a fases clínicas, la evaluación de inmunogenicidad y eficacia es un aspecto esencial para la evaluación de candidatos a vacuna.

Palabras clave: malaria, vacunas, inmunogenicidad vacunal, Plasmodium falciparum, eficacia, experimentación animal.

1 Universidad de Boyacá, Tunja, Colombia.

2 Universidad del Rosario, Bogotá, Colombia

Correspodencia: Anny Jineth Camargo-Mancipe Dirección Carrera 2a este № 64-169. Tunja, Colombia. Celular: 3204258352

Correo electrónico: anncamargo@uniboyaca

Citar este artículo así:

Camargo-Mancipe AJ, Díaz-Arévalo D, Salamanca- Jiménez DR, Cuy-Chaparro LE, Camargo-Mancipe DF. Revisión de estudios pre-clínicos de candidatos a vacuna contra la malaria causada por Plasmodium falciparum. Revista Investig Salud Univ Boyacá. 2019;6(2): 200-223. doi: https:// doi.org/10.24267/23897325.353 


\title{
Review of preclinical studies of candidates for malaria vaccine caused by Plasmodium falciparum
}

\begin{abstract}
SUMMARY
Introduction. Malaria disease causes approximately 400,000 deaths by year, especially in children under 5 years, the search for an effective and safe vaccine, remains to be a challenge for researchers, however before starting the clinical phase studies, preclinical trials in animal models should provide safety and immunogenicity results that lead to effective protective responses.

Objective. To review the main characteristics of the immune response and efficacy in pre-clinical studies of candidates for vaccine against malaria by Plasmodium falciparum.

Methods. A descriptive review of the main preclinical studies of malaria vaccine candidates, based on subunits, attenuated parasites and multi-stage, multi-epitope vaccines, which have been carried out to evaluate immunogenicity and efficacy, is presented. This review was carried out based on the search of literature in electronic databases specialized in scientific research. 118 documents were found, of which 91 were selected and 17 were excluded because they did not meet the inclusion criteria, for a total of 74 references analyzed.
\end{abstract}

Results. Many candidates for malaria vaccine caused by Plasmodium falciparum have reported promising results against homologous strains, however, given the challenge with heterologous strains, efficacy decreases, on the other hand, the lasting immune and protective response continues to be a key objective, becoming a priority.

Conclusions. Preclinical studies in animal models are necessary before advancing to clinical phases, the evaluation of immunogenicity and efficacy is an essential aspect for the evaluation of vaccine candidates.

Keywords: malaria, vaccines, immunogenicity, vaccine, Plasmodium falciparum, efficacy, animal experimentation. 
Anny Jineth Camargo-Mancipe, Diana Díaz-Arévalo, David Ricardo Salamanca- Jiménez,

Laura Esperanza Cuy-Chaparro, Diego Fernando Camargo-Mancipe

\title{
Revisão de estudos pré-clínicos de candidatos á vacina contra a malária causados por Plasmodium falciparum
}

\begin{abstract}
RESUMO
Introdução: A malária é uma doença que causa aproximadamente 400.000 óbitos por ano, principalmente em crianças menores de 5 anos, a procura por uma vacina eficaz e segura, continua sendo um desafio para os pesquisadores, porém, antes de iniciar os estudos de fase clínica, os testes pré-clínicos no modelo animal devem fornecer resultados de segurança e imunogenicidade que levam a respostas efetivas de proteção.
\end{abstract}

Objetivo: Verificar as principais características da resposta imune e eficácia em estudos pré-clínicos de candidatos à vacina contra a malária por Plasmodium falciparum.

Métodos: Revisão descritiva dos principais estudos pré-clínicos de candidatos à vacina contra a malária, com base em subunidades, parasitas atenuados e vacinas de vários estágios e multiepítopo, que foram realizadas para avaliar a imunogenicidade e eficácia em modelos animais. Esta revisão foi realizada com base na pesquisa de literatura em bases de dados eletrônicas especializadas em pesquisa científica. Foram encontrados 118 documentos, dos quais 91 foram selecionados e 17 foram excluídos por não atenderem aos critérios de inclusão, para um total de 74 referências analisadas.

Resultados: Muitos candidatos à vacina contra a malária causada por Plasmodium falciparum relataram resultados promissores contra cepas homólogas, no entanto, diante do desafio com cepas heterólogas a eficácia diminui, por outro lado, a resposta imune e protetora duradoura continua sendo um objetivo fundamental, tornando-se uma prioridade.

Conclusões: Estudos pré-clínicos em modelo animal são necessários antes de passar para as fases clínicas, a avaliação da imunogenicidade e eficácia é um aspecto essencial para a avaliação dos candidatos a vacina.

Palavras-chaves: Malária, vacinas, imunogenicidade vacinal, Plasmodium falciparum, eficácia, experimentação animal. 


\section{INTRODUCCIÓN}

La malaria es una enfermedad potencialmente mortal; cinco especies de Plasmodium pueden afectar al ser humano, siendo los más prevalentes Plasmodium falciparum (P. falciparum) y Plasmodium vivax ( $P$. vivax), el ciclo de vida de estos parásitos comienza cuando son transmitidos al ser humano por la picadura de mosquitos hembra del género Anopheles, (1-5) (Figura 1).Entre las proteínas que están involucradas en el proceso de invasión se resaltan: proteína de circumsporozoíto - 1 (CSP-1), proteína anónima relacionada con la trombospondina (TRAP), proteínas de superficie del merozoíto (MSP-1, MSP-2, MSP-4, MSP-7, MSP-9, MSP-10), antígeno de unión al eritrocito (EBA-175) y proteína de $P$. falciparum homóloga a la de unión al reticulocito (PfRh5) $(6,7)$.

Esta enfermedad representa uno de los mayores problemas de salud pública a nivel mundial; en 2017 se reportaron aproximadamente 219 millones casos de malaria, con un estimado de 435.000 muertes. Los niños menores de 5 años fueron los más vulnerables, quienes representaron el $61 \%$ (266.000) de todas las muertes por esta enfermedad durante el 2017. Del total de casos, la mayoría ocurrieron en países africanos (92\%) (8).

Se espera que para el 2030 la incidencia y la mortalidad por malaria se reduzca mínimo en un 90\% y sea eliminada al menos en 35 países; entre las principales medidas para reducir la transmisión de la malaria se encuentra la lucha contra los vectores, por lo tanto, para proteger a toda la población que se encuentra en riesgo de infectarse se recomienda el uso de mosquiteros, así como la fumigación de interiores con insecticidas de acción residual. Adicionalmente, las personas que viajen a zonas endémicas deben utilizar antimaláricos de manera profiláctica (sulfadoxina-pirimetamina) (8).

Pese a estas medidas, la enfermedad continúa siendo de difícil control, situación que lleva a que muchas instituciones científicas trabajen en el desarrollo de una vacuna, candidata efectiva contra la malaria, que permita erradicar la enfermedad mediante el abordaje de diferentes estrategias como vacunas de subunidades, vacunas con parásitos completos atenuados y vacunas con vectores virales (7-10).

Como requisito de candidatos a vacuna, las autoridades regulatorias internacionales como la FDA (Food and Drug Administration), ICH (International Conference on Harmonisation), la EMA (European medicines Agency) y la OMS, indican que se deben realizar estudios preclínicos que permitan confirmar la potencia, inocuidad, inmunogenicidad y seguridad, lo que permitirá iniciar los estudios de fase clínica en humanos (9).

A través de la historia, diversos candidatos a vacuna han sido evaluados en primates no 
Anny Jineth Camargo-Mancipe, Diana Díaz-Arévalo, David Ricardo Salamanca- Jiménez, Laura Esperanza Cuy-Chaparro, Diego Fernando Camargo-Mancipe

Figura 1. Título: Ciclo de vida Plasmodium falciparum.

\section{b. Esporozoitos}

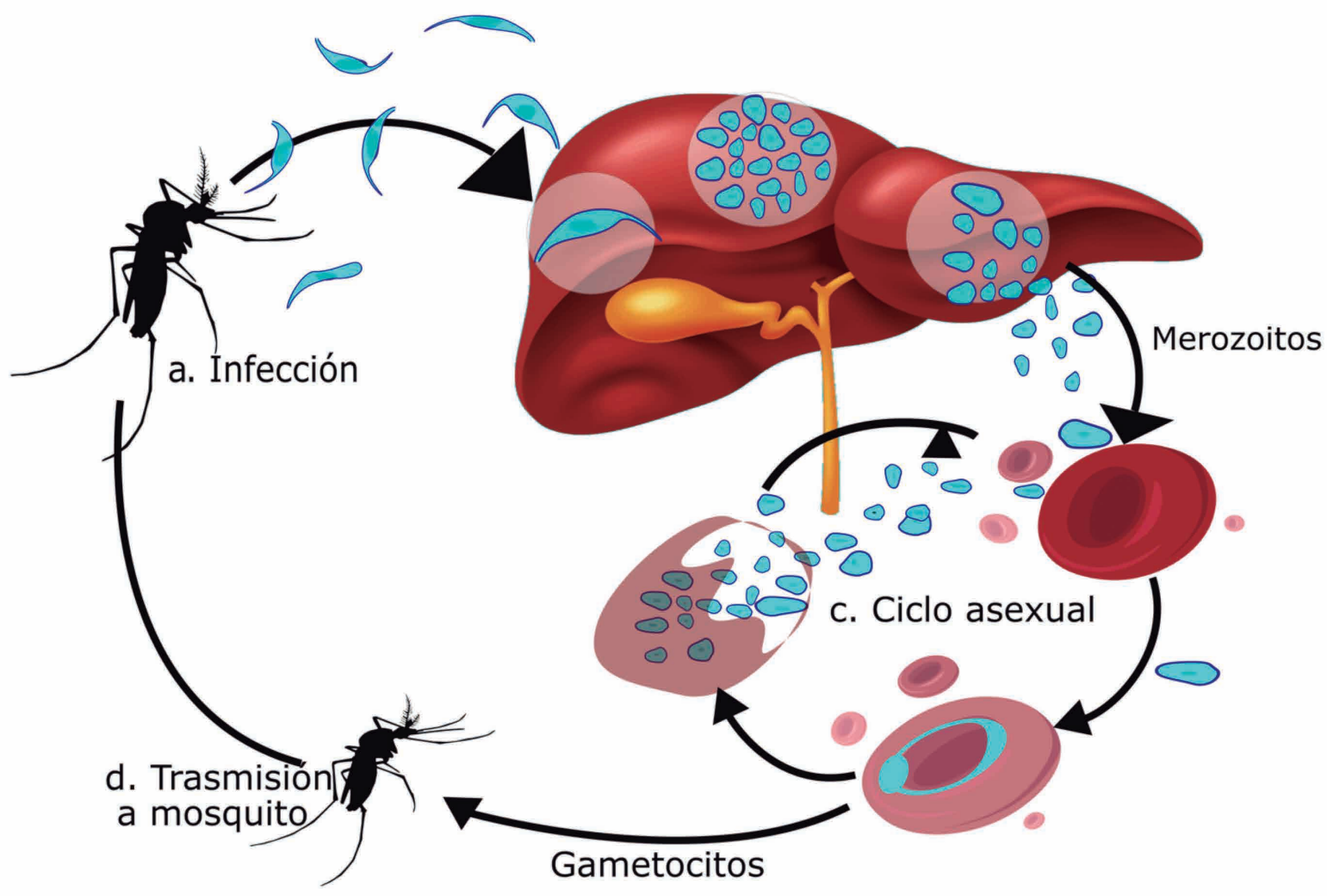

a. Transmisión del parásito al hospedero humano por medio de la picadura del mosquito hembra Anopheles. b. Invasión de los esporozoítos a las células hepáticas. c. La ruptura de los esporozoítos libera miles de merozoítos que invaden los glóbulos rojos (ciclo asexual) d. Algunos parásitos se pueden diferenciar en gametocitos, los cuales son ingeridos por los mosquitos hembras Anopheles (ciclo sexual).

Fuente: Elaboración de los autores. 
humanos, como por ejemplo los del género Aotus spp. (11), esto se debe a que son altamente susceptibles a la malaria humana y sus moléculas del sistema inmune tienen una alta identidad (80 - 100\%) con las de los humanos (12-18), por ello, se consideran un buen modelo experimental para evaluar la respuesta inmunológica de candidatos a vacuna para el control de malaria (19-20); sin embargo, teniendo en cuenta las consideraciones éticas y la dificultad de acceso al uso de este modelo primate no humano, también se usan otros modelos animales como los roedores para la evaluación de seguridad, inmunogenicidad y eficacia, así mismo, el auge de ratones humanizados ha permitido el estudio directo de parásitos de la malaria humana in vivo en el laboratorio (21).

Teniendo en cuenta la importancia de los estudios preclínicos que permiten garantizar la seguridad de cualquier formulación y avanzar a fases clínicas en humanos, el objetivo de este artículo es revisar los datos de formulación, seguridad, inmunogenicidad y eficacia de los principales ensayos preclínicos en modelo animal de candidatos a vacuna contra la malaria causada por $P$. falciparum.

\section{METODOLOGÍA}

Se realizó una revisión descriptiva sobre los principales candidatos a vacuna contra la malaria basados en subunidades mínimas, parásitos vivos atenuados y vacunas multi-estadio, multi-epitope.
La búsqueda de la literatura científica se efectuó a partir de artículos publicados en las bases de datos electrónicas como PubMed, WEB OF SCIENCE, Embase, LILACS, REDALYC y se realizaron búsquedas secundarias en las listas de referencias bibliográficas de los artículos identificados.

Las palabras claves se validaron mediante el descriptor Medical Subject Headings (MeSH) en idioma inglés: malaria, vaccines, Immunogenicity, vaccine, Plasmodium falciparum, efficacy, animal experimentation.

Fueron seleccionados todos los artículos en el idioma inglés y español, publicados entre 1967 y 2019 que describen los primeros estudios preclínicos, los cuales fueron pioneros para el desarrollo de vacunas contra la malaria, así como recientes artículos con los últimos avances en vacunas. Se encontraron 118 artículos, de los cuales se seleccionaron 91 por título, materiales y métodos y resumen; de estos, se excluyeron 17 por no presentar resultados de eficacia e inmunogenicidad de estudios preclínicos en modelo animal. En total, se analizaron 74 referencias que presentaban resultados detallados.

\section{DESARROLLO Y DISCUSIÓN}

\section{Candidatos a vacuna contra la malaria fase pre clínica}


Los esfuerzos para obtener una vacuna totalmente efectiva contra la malaria siguen en curso. Un primer paso para su desarrollo incluye la evaluación de la seguridad e inmunogenicidad en un modelo animal, en el cual se garantice la seguridad de los componentes de la formulación, dosis y vía de administración, para que así, sea posible avanzar a los estudios de fase clínica en seres humanos.

Estudios preclínicos pioneros en el desarrollo de una vacuna contra la malaria, realizados por Nussenzweig y colaboradores, demostraron los efectos generados por la radiación sobre los Spz y la protección que estos indujeron en ratones retados con P. berghei (22-26).

Sobre estos hallazgos se han logrado diversos avances científicos como la comprensión de mecanismos moleculares y celulares del parásito implicados en la invasión a la célula hospedera, permitiendo la evaluación de un sin número de candidatos a vacuna contra la malaria (1).

En esta revisión se tratan las principales vacunas basadas en subunidades que fueron evaluadas en estudios preclínicos como EBA-175, PfRH5, AMA1-RON2L, MSP1, y RTS, S/AS01; esta última, es la vacuna más ampliamente probada en seres humanos e incluye la proteína CSP de $P$. falciparum (PfCSP), ubicada en la superficie del Spz. Adicionalmente, se discuten los resultados de otras estrategias como el uso de parásitos atenuados, cuyo candidato más avanzado es PfSPZ fabricado por la compañía Sanaria inc., a partir de Spz vivos atenuados y asépticos de la cepa $P$. falciparum NF54 (27).

Numerosos estudios se han realizado con estos candidatos a vacuna, sin embargo, la alta complejidad del parásito, su polimorfismo y la gran variedad genética del hospedero han dificultado el desarrollo de una estrategia totalmente efectiva, por lo tanto, en la presente revisión se analiza una metodología lógica y racional basada en la producción de una vacuna multi-epitope, multi-estadio, químicamente sintetizada y basada en mínimas subunidades que incluye las principales regiones de las proteínas de $P$. falciparum involucradas en el proceso de invasión $(1,28)$.

\section{Vacunas basadas en subunidades}

\section{EBA 175}

EBA-175 es una proteína de $175 \mathrm{kD}$ de $P$. falciparum involucrada en la unión a eritrocitos (29), conformada por una región rica en cisteína en el N-terminal de 616 aminoácidos Ilamada región II, altamente conservada, que contiene el dominio de unión a su receptor glicoforina A (30).

En un estudio se evaluó la inmunogenicidad y eficacia protectora de: a) una vacuna de ADN plasmídico que codifica la región II de la proteína EBA-175 
de $P$ falciparum (Dv-Dv), b) una vacuna recombinante en adyuvante (Pv-Pv) y c) una vacuna de ADN con una dosis de proteína recombinante (Dv-Pv), en 34 monos Aotus spp. Después de tres dosis de inmunización los títulos de anticuerpos (Acs) fueron mayores $(342,092-2,228,781)$ en monos del grupo 6, inmunizados con la vacuna basada en la proteína recombinante, comparado con los títulos de Acs $(1,761$ - 54,455) obtenidos en animales del grupo 1 inmunizados con ADN. Cuatro semanas después de la última inmunización, los animales fueron retados con $10^{4} \mathrm{GR}$ infectados
P. falciparum, a pesar de que todos los monos presentaron parasitemia, los niveles fueron más bajos en los monos del grupo 6 que recibieron plásmidos o proteínas que contenían la región II de EBA-75 comparado con los controles en los que los títulos de Acs fueron cero y las parasitemias alcanzaron niveles elevados entre 319,077 - 880,279 parásitos/ $\mu \mathrm{L}$ (Tabla1.). Estos resultados demostraron que la región II de la proteína EBA-175 induce Acs con un efecto antiparasitario significativo (31).

Tabla 1. Vacuna EBA-175: Títulos de Acs cuantificados por ELISA en 34 monos Aotus previo al desafío con parásitos (pre-reto) y nivel de parasitemia detectado posterior a este reto.

\begin{tabular}{|c|c|c|c|c|c|}
\hline Referencia & Animales & Controles & $\begin{array}{l}\text { Inmunización con vacuna } \\
(\text { semana } 0,4,8, \text { y } 24)\end{array}$ & $\begin{array}{c}\text { Títulos de Acs (ELISA) } \\
\text { Pre-reto }\end{array}$ & $\begin{array}{l}\text { Máximo No. } \\
\text { Parásitos/ } \mu \mathrm{L}\end{array}$ \\
\hline \multirow{8}{*}{$\begin{array}{l}\text { Jones, T. R. et } \\
\text { al., 2001. (31) }\end{array}$} & \multirow{8}{*}{$\begin{array}{l}34 \text { monos Aotus } \\
\text { nancymaae }\end{array}$} & & $\begin{array}{c}\text { Grupo } 1(\mathrm{n}=4): 4 \text { dosis } \\
\text { Dv-Dv }\end{array}$ & $1,761-54,455$ & $33,861-491,060$ \\
\hline & & & $\begin{array}{c}\text { Group } 2(\mathrm{n}=4): 4 \text { dosis } \\
\text { Dv-Pv }\end{array}$ & $295,941-952,841$ & $96,366-313,603$ \\
\hline & & & $\begin{array}{c}\text { Grupo } 3(\mathrm{n}=4): 4 \text { dosis } \\
\text { Dv-DvPv }\end{array}$ & $112,838-302,896$ & $265,039-752,470$ \\
\hline & & & $\begin{array}{c}\text { Grupo } 4(\mathrm{n}=4): 4 \text { dosis } \\
\text { Dc-Dc }\end{array}$ & $0-1,592$ & $422,129-749,924$ \\
\hline & & & $\begin{array}{c}\text { Grupo } 6(\mathrm{n}=4): 4 \text { dosis } \\
\mathrm{Pv}-\mathrm{Pv}\end{array}$ & $342,092-2,228,781$ & $17,335-313,561$ \\
\hline & & $\begin{array}{l}\text { Control }(n=4) \text { : } \\
\text { Dc-DcPc }\end{array}$ & & 0 & $304,438-467,318$ \\
\hline & & $\begin{array}{l}\text { Control adyuvante } \\
\quad(n=4): \text { Pc-Pc }\end{array}$ & & 0 & $193,369-652,158$ \\
\hline & & Controles $(n=4): n$ & & 0 & $319,077-880,279$ \\
\hline
\end{tabular}

Tabla 1. Vacuna EBA-175. Dc, Vacuna control ADN; Dv, Vacuna ADN EBA de P. falciparum; Pc, adyuvante control; $\mathbf{n}$, nada; Pv, Vacuna proteína recombinante EBA de P. falciparum. *Los títulos de Acs son basados en 4 individuos. 
Anny Jineth Camargo-Mancipe, Diana Díaz-Arévalo, David Ricardo Salamanca- Jiménez,

Laura Esperanza Cuy-Chaparro, Diego Fernando Camargo-Mancipe

\section{Proteína de P. falciparum homóloga a la de unión al reticulocito (PfRH5)}

En varios estudios se encontró que la interacción entre proteínas del complejo PfRH5-PfRipr-CyRPA $y$ el receptor basigina, cumplen un rol importante durante la invasión de mrz de $P$. falciparum a los eritrocitos humanos (32) y que los Acs humanos anti- PfRH5 purificados por afinidad inhiben la invasión del parásitos in vitro $(33,34)$ but the relevance of naturally acquired PfRH5-specific antibodies in humans is unclear. InMethods. We assessed pre-malaria season PfRH5-specific immunoglobulin G (IgG. Además, la caracterización de su estructura tridimensional, ha permitido identificar la función crítica de esta proteína, convirtiendo a PfRH5 en un posible candidato a vacuna (35).

En un estudio preclínico se evaluó la inmunogenicidad en 31 monos Aotus nancymaae distribuidos aleatoriamente en 5 grupos, inmunizados con diferentes formulaciones: a) vectores virales de adenovirus/poxvirus previamente probados $(36,37)$ o b) la proteína RH5 emulsificada en adyuvante completo/incompleto de Freund (38). Las inmunizaciones se realizaron vía intramuscular (IM) los días 0 y 54, con excepción de las que contenían adyuvante de Freud, que se administraron por vía subcutánea (SC) los días 0, 26 y 54. La eficacia se probó 15 días después de la última inmunización mediante un reto heterólogo con 104 GR infectados de la cepa FVO de P. falciparum.
Los animales inmunizados con la proteína PfRH5 emulsionada en adyuvante de Freund (Grupo B) tuvieron altas concentraciones de Acs $(700 \mu \mathrm{g} /$ $\mathrm{ml}$ ) específicos de PfRH5 y una eficacia protectiva significativa: dos animales permanecieron en periodo sub-patente y un animal con un solo parásito visto en una ocasión, los tres animales restantes controlaron la parasitemia alrededor de los 30 días de observación, mientras que en los demás grupos inmunizados con MVA o Chad63 en combinación con PfRH5 o PfAMA1 (Grupos C, $D$ y E) las concentraciones de Acs fueron inferiores y oscilaron entre $54 \mu \mathrm{g} / \mathrm{ml}$ y $320 \mu \mathrm{g} / \mathrm{ml}$ (Tabla 2.) (39). Con estos resultados se respalda PfRH5 como un candidato a vacuna prometedor, sin embargo, se deben optimizar las formulaciones de esta vacuna para lograr obtener altas respuestas de Acs protectivos.

\section{Antígeno apical de membrana 1 - Proteína del cuello de roptrias 2 (AMA1-RON2L)}

La proteína AMA1 está conformada por tres dominios, el dominio I en la región $\mathrm{N}$-terminal (residuos 97-307), el dominio II desde los residuos 309 hasta 435 y el dominio III, más conservado, localizado en el C-terminal (residuos 436-546); este último es esencial para la invasión de $P$. falciparum. AMA1 que inicialmente es almacenada en los micronemas y luego es traslocada a la superficie del mrz, donde forma un complejo con RON2 del cual se ha reportado que contiene tres hélices 
Tabla 2. Vacuna PfRH5: Concentraciones de anticuerpos específicos para la proteína PfRH5 en 31 monos Aotus inmunizados con PfRH5 emulsionada en adyuvante de Freund, MVA o Chad63 en combinación con PfRH5 O PfAMA1.

\begin{tabular}{|c|c|c|c|c|}
\hline Referencias & Animales & Controles & Inmunización & $\begin{array}{l}\text { Concentraciones de Acs } \\
\text { específicas de PfRH5 }\end{array}$ \\
\hline \multirow{5}{*}{$\begin{array}{l}\text { Douglas, A.D. et } \\
\text { al., 2015. (39) }\end{array}$} & \multirow{5}{*}{$\begin{array}{l}31 \text { monos Aotus } \\
\text { nancymaae }\end{array}$} & $\begin{array}{c}\text { Grupo A }(\mathrm{n}=6) \text { : } \\
\text { día 0 Chad63-Rluc, día } 54 \\
\text { PBS-Abisco-100. }\end{array}$ & & --- \\
\hline & & & $\begin{array}{c}\text { Grupo B }(\mathrm{n}=6) \text { : } \\
\text { día } 0 \text { PfRH5-CFA, } \\
\text { día } 26 \text { y } 54 \text { PfRH5-IFA. }\end{array}$ & $700 \mu \mathrm{g} / \mathrm{ml}$ \\
\hline & & & $\begin{array}{c}\text { Grupo C (n=7): } \\
\text { día 0 Chad63- PfRH5, día } 54 \\
\text { PfRH5-Abisco 100. } \\
\end{array}$ & $54 \mu \mathrm{g} / \mathrm{ml}$ \\
\hline & & & $\begin{array}{c}\text { Grupo D (n=6): } \\
\text { día 0 Chad63- PfRH5, día } 54 \\
\text { MVA-PfRH5. }\end{array}$ & $320 \mu \mathrm{g} / \mathrm{ml}$ \\
\hline & & & $\begin{array}{c}\text { Grupo E }(\mathrm{n}=6) \text { : } \\
\text { día 0 Chad63-PfAMA1, día } 54 \\
\text { PfAMA1-Abisco } 100 .\end{array}$ & $140 \mu \mathrm{g} / \mathrm{ml}$ \\
\hline
\end{tabular}

Tabla 2. Vacuna PfRH5. RLuc: Renilla luciferasa, CFA: Adyuvante de Freud completo, CFI: Adyuvante de Freud incompleto. MVA: del inglés, modified vaccinia virus Ankara, Chad63: chimpanzee adenovirus.

hidrófobas, cuyos dos residuos Cys conservados, presentes entre la segunda y la tercera hélices hidrófobas denominadas RON2L, son esenciales para la unión a AMA1 (40-42). Esta proteína es un candidato a vacuna, sin embargo, a pesar de mostrar ser segura ha fallado en cuanto a la producción de Acs protectores funcionales $(43,44)$. Teniendo en cuenta estos resultados y con el fin de obtener una mayor eficacia, se evaluó el complejo PfAMA1-RON2L en
22 Aotus nancymaae mediante un reto homólogo con la cepa virulenta FVO de $P$. falciparum. Los monos fueron vacunados con 3 dosis de la formulación AMA1, AMA1-RON2L o control, 4 semanas después de la última inmunización; el reto se realizó con $10^{4}$ eritrocitos infectados por vía intravenosa. Los animales inmunizados con AMA 1 se infectaron, mientras que el $50 \%$ de los animales inmunizados con el complejo AMA1- 
RON2L no presentaron parasitemia hasta el final del estudio (40 días después del reto) y tuvieron respuestas de Acs con mayor capacidad de neutralización en comparación con el grupo vacunado solo con AMA1. En este estudio se destaca la capacidad del complejo AMA1-RON2L para proteger ante el reto homólogo con una eficacia $87.5 \%$

(Tabla 3.) e inducir Acs bloqueantes contra epítopos conservados que se correlacionan con neutralización de la invasión; igualmente, se resalta la importancia de realizar estudios futuros que permitan identificar epítopos importantes que sean objetivo de Acs protectores (45).

\section{Proteína quimérica de superficie de merozoíto (MSP1)}

En un estudio se evaluó la inmunogenicidad de una vacuna quimérica contra la malaria, la cual incluía epítopos de células B neutralizantes de la proteína MSP1 de $P$. falciparum (rPf MSP1 ${ }_{19}$ ), genéticamente ligados a una proteína de superficie de merozoíto de $P$. falciparum, altamente inmunogénica, y a la proteína de superficie de merozoíto 8 (MSP8) ((rPfMSP8 ( $\triangle$ Asn/Asp)). Para la evaluación de inmunogenicidad y eficacia se utilizaron 18 monos Aotus adultos, los cuales fueron divididos al azar en un grupo vacunado que recibió $50 \mu \mathrm{g}$ de rPfMSP1 / 8, otro grupo que recibió $r P f M S P 8$ ( $\triangle$ Asn/Asp) emulsionado en Montanide ISA 720 y un grupo control inmunizado con solución salina o Montanide solo, en un esquema de inmunización 0,28 y 84 días. Los epítopes Pf $\mathrm{MSP}_{19}$ y la vacuna con $r$ Pf MSP8 $(\triangle A s n / A s p)$ no generaron títulos significativos de Acs contra $P$. falciparum después de la primera y segunda inmunización, sin embargo, rPf MSP1/8 indujo una respuesta alta de Acs (títulos IgG específicos de antígeno >1.0E +05 determinados por ELISA), así como una inhibición

Tabla 3. Vacuna AMA1-RON2L: Porcentaje de eficacia en monos Aotus inmunizados con AMA 1, AMA1-RON2L o grupo control.

\begin{tabular}{|c|c|c|c|c|}
\hline Referencia & Animales & Controles & Inmunización (3 dosis) & Eficacia \\
\hline \multirow{3}{*}{$\begin{array}{c}\text { Srinivasan, P. et al., } 2017 . \\
\text { (45). }\end{array}$} & \multirow{3}{*}{$\begin{array}{l}22 \text { monos Aotus nan- } \\
\text { cymaae }\end{array}$} & $\begin{array}{l}\text { Grupo } 1(n=6) \text { : Con- } \\
\text { trol con adyuvante }\end{array}$ & & $-\cdots$ \\
\hline & & & Grupo $2(n=8)$ : solo AMA1 & $25 \%$ \\
\hline & & & $\begin{array}{c}\text { Grupo } 3(\mathrm{n}=8) \text { : Complejo AMA1- } \\
\text { RON2L }\end{array}$ & $87.5 \%$ \\
\hline
\end{tabular}

Vacuna AMA1-RON2L AMA1: Antígeno apical de membrana 1, RON2L: Proteína de cuello de roptrias 2. Acs: Ancticuerpos 
eficaz del crecimiento del parásito comparado con los otros grupos. Una vez realizado el reto con FVO, tres animales inmunizados con MSP1/ 8 controlaron la parasitemia, dos de los cuales eliminaron su infección y uno requirió tratamiento farmacológico debido a la anemia. Estos datos demostraron que la vacuna Pf MSP1/8 es segura e induce Acs que inhiben el crecimiento de $P$. falciparum apoyando su uso como un componente de una vacuna multivalente para su evaluación en humanos (46).

\section{RTS,S.}

Por otra parte, están los estudios realizados con RTS,S una vacuna compuesta por una región repetitiva en tandem NANP de la proteína CSP (R), un C-terminal con epítopos de células $\mathrm{T}(\mathrm{T})$, fusionados al antígeno de superficie (S) de la hepatitis $B(\mathrm{AgSHB})$, expresadas en células de levadura $\mathrm{S}$. cerevisiae que actualmente se encuentra en estudios clínicos fase III $(47,48)$; esta vacuna pre-eritrocítica se dirige a un gran segmento (aminoácidos 207 a 395) de la CSP de P. falciparum donde se han identificado un sin número de epítopos variables $(49,50)$. En su fase preclínica, fue evaluada en modelo primate no humano con diferentes adyuvantes como ASO2A (51), además de la combinación con la proteína TRAP (RTS,S+TRAP/ ASO2A) (52), demostrando que la formulación era segura y eficaz en modelo animal (51), lo que permitió iniciar los estudios clínicos. Sin embargo, con el fin de encontrar una mayor eficacia lograda por
RTS, S, muchas estrategias han sido usadas como nuevos adyuvantes $(47,53,54)$, vectores virales (55-57) o en combinación con otros antígenos protectivos (51), por lo cual se evaluó la seguridad e inmunogenicidad de RTS,S, MSP142 y AMA1 formulada con el adyuvante ASO2A en monos Rhesus. Un total de 48 monos Rhesus fueron seleccionados y distribuidos en 7 grupos, e inmunizados en un esquema de 3 dosis, solo o combinado AMA1 con MSP142 y/o RTS,S y un grupo control que recibió solución salina. La respuesta de Acs anti-CSP con la inmunización usando solo RTS,S (Títulos geométricos: 6,368) fue estadísticamente similar a la obtenida con RTS,S + AMA1 (Títulos geométricos: 13,933) y RTS,S en combinación con AMA1 + MSP142 (Títulos geométri$\cos 8$,991) (Tabla 4). En los ensayos de inhibición de crecimiento in-vitro, MSP142 tuvo una baja inhibición del crecimiento del parásito in vitro (\% media de inhibición $=10.1$ ), RTS,S sola mostró una media inhibitoria de $21 \%$, mientras que AMA1 tuvo una media de inhibición del $52.7 \%$ (51). Los resultados de este estudio sugieren que AMA1, MSP142 y RTS,S tienen perfiles de reactogenicidad y seguridad aceptables, sin embargo, la combinación de AMA1 con uno de los dos antígenos redujo las respuestas de Acs y MSP142 disminuyó significativamente las respuestas de IFN e IL-5 específicas de AMA1 lo que generó una interferencia inmune, que debe ser considerada para el desarrollo de vacunas contra la malaria basadas en múltiples etapas del parásito (51). 
Anny Jineth Camargo-Mancipe, Diana Díaz-Arévalo, David Ricardo Salamanca- Jiménez,

Laura Esperanza Cuy-Chaparro, Diego Fernando Camargo-Mancipe

Tabla 4. Vacuna RTS,S: Títulos medios geométricos cuantificados por ELISA en 48 monos Macacos Rhesus inmunizados con diferentes formulaciones de AMA1 - MSP142 - RTS,S o grupo control.

\begin{tabular}{|c|c|c|c|c|}
\hline Referencias & Animales & Control & $\begin{array}{l}\text { Inmunización en } 0.5 \mathrm{ml} \text { de adyuvante } \\
\text { AS02A (Semana } 0,4 \text { y } 8 \text { ) }\end{array}$ & $\begin{array}{c}\text { Títulos medios } \\
\text { geométricos ELISA }\end{array}$ \\
\hline \multirow{7}{*}{$\begin{array}{l}\text { Pichyangkul, S. et } \\
\text { al., 2009. }\end{array}$} & \multirow{7}{*}{$\begin{array}{l}48 \text { Macacos } \\
\text { Rhesus. }\end{array}$} & & Grupo $1(n=7)$ : AMA1 solo & 26,357 \\
\hline & & & Grupo $2(\mathrm{n}=7): \mathrm{MSP}_{142}$ & 38,104 \\
\hline & & & Grupo 3 (n=7): RTS,S & 6,368 \\
\hline & & & Grupo $4(\mathrm{n}=7)$ : AMA1 $+\mathrm{MSP}_{142}$ & 8,911 \\
\hline & & & Grupo 5 (n=7): AMA1+ RTS,S & 13,933 \\
\hline & & & Grupo $6(\mathrm{n}=7)$ : AMA1+MSP ${ }_{142}+\mathrm{RTS}, \mathrm{S}$ & 8,991 \\
\hline & & $\begin{array}{l}\text { Grupo } 7(\mathrm{n}=6) \text { Control } \\
\text { Solución salina }\end{array}$ & & --- \\
\hline
\end{tabular}

Vacuna RTS, S. AMA1: Antígeno apical de membrana 1, MSP $_{142}$ : Proteína quimérica de superficie de merozoíto.

\section{Vacunas con parásitos completos atenuados}

\section{PfSPZ}

Las vacunas con parásitos atenuados contienen todos los antígenos del parásito, este enfoque permite inducir diferentes respuestas inmunes. Los primeros avances con este tipo de vacunas fueron en 1967 donde el grupo Nussenzweig y colaboradores, usando Spz de P. berghei irradiados e inoculados en roedores, demostraron que estos Spz metabólicamente activos y móviles, invadían hepatocitos, pero no podían convertirse en trofozoitos o esquizontes hepáticos (22). Posteriormente, en la inmunización de monos Rhesus con Spz de $P$. knowlesi irradiados, los niveles de Acs fueron asociados con protección (58). Estos Spz irradiados se probaron en un estudio de fase clínica I, en el cual 11 voluntarios fueron inmunizados con más de mil picaduras de mosquitos irradiados que transmitían Spz de la cepa NF54 de $P$. falciparum o el clon 3D7/NF54. Todos los participantes 2-4 semanas se protegieron ante el reto homólogo, sin embargo, cuando el reto fue realizado con una cepa heteróloga (7G8), solo $2 / 10$ voluntarios se protegieron (59). A pesar de esto, se consideró que la vía de inmunización a través de las picaduras de mosquitos no fue la más adecuada. 
Estudios adicionales demostraron que la vía de inoculación intravenosa fue crítica para inducir células T productoras de IFN- $\gamma$ CD8 (+) específicas para PfSPZ en el hígado comparado con la vía subcutánea (27).

Con los resultados hasta hoy obtenidos de diversos ensayos preclínicos llevados a cabo, y un sin número de ensayos clínicos, se espera que a principios de 2020 los investigadores realicen un ensayo que involucrará a 2.100 personas de 2 a 50 años, lo que permitirá proporcionar datos de eficacia y seguridad necesarios para la aprobación regulatoria de esta vacuna, según lo referido por el investigador de malaria Steven Hoffman, quien lidera el estudio y es el director ejecutivo de Sanaria, la compañía en Rockville, Maryland, que desarrolló la vacuna.

\section{Vacunas muti-epítope, multi-estadio}

\section{SPf66}

La Fundación Instituto de Inmunología (FIDIC) lleva más de 30 años trabajado en el desarrollo de una vacuna para control de la malaria. En una primera aproximación, se llevó a cabo el estudio preclínico en Aotus trivirgatus, los cuales fueron inmunizados con péptidos provenientes de las proteínas MSP1, RESA y la región repetitiva de CSP (NANP); cada mono recibió cinco inmunizaciones $(0,30,45,60$ y 75 días); quince días después de la última inmunización, los monos fueron retados con $P$. falciparum (cepa FVO). Quince de los dieciocho péptidos usados en esta inmunización, indujeron respuesta de Acs contra esquizontes de $P$. falciparum, algunos de los quince péptidos mostraron un retraso significativo en la parasitemia sugiriendo su habilidad para inducir protección parcial, por lo cual se inmunizó un nuevo grupo de monos con combinaciones de 2 o 3 péptidos (Tabla 5.). Usando el mismo esquema de inmunización, se encontró que los monos tuvieron altos títulos de anticuerpos (Títulos IFI: $\sim 40$ - 160); en este nuevo grupo, la mayoría de los animales desarrollaron parasitemia baja y se curaron espontáneamente, los otros monos no mostraron signos de enfermedad, tampoco se detectaron parásitos en sangre hasta los 180 días de seguimiento con dos de los péptidos derivados del estadio de merozoíto (MSP1 RESA) y la región de repeticiones NANP de la proteína CSP del estadio de esporozoíto. Con estos tres péptidos se sintetizó una vacuna quimérica denominada SPf66 que mostró una alta eficacia protectiva, puesto que $3 / 6$ monos inmunizados desarrollaron baja infección con una parasitemia máxima de $5 \%$ y el resto fueron protegidos; estos óptimos resultados permitieron avanzar a estudios de fase clínica en humanos (60). Diversos estudios clínicos realizados en Colombia, Ecuador, Venezuela, Brasil y Tanzania mostraron una eficacia que oscilaba entre el $23 \%$ y el $66 \%(61-64)$. A pesar de mostrar ser eficaz en retos heterólogos y de haberse mantenido a la fecha como la vacuna 
Anny Jineth Camargo-Mancipe, Diana Díaz-Arévalo, David Ricardo Salamanca- Jiménez,

Laura Esperanza Cuy-Chaparro, Diego Fernando Camargo-Mancipe

Tabla 5. Vacuna SPf66: Títulos IFI obtenidos en monos Aotus posterior a la inmunización con los péptidos provenientes de las proteínas MSP1, RESA y la región de repeticiones NANP de CSP de P. falciparum.

\begin{tabular}{|c|c|c|c|}
\hline Referencias & Animales & Inmunización (Día 0, 30, 45, 60 y 75) & Títulos IFI \\
\hline $\begin{array}{l}\text { Manuel E. Pata- } \\
\text { rroyo. et al., } 1987 \\
\text { (60) }\end{array}$ & $\begin{array}{l}\text { Grupos de } 4 \text { a } 6 \\
\text { Aotus trivirgatus }\end{array}$ & $\begin{array}{c}\text { Péptido } 35.1+55.1+83.1 \text { derivados del estadio de merozoíto (MSP1 RESA) } \\
\text { y la región de repeticiones NANP de la proteína CSP del estadio de esporo- } \\
\text { zoíto. }\end{array}$ & $\sim 40-160$ \\
\hline
\end{tabular}

Vacuna SPf66. IIFI: ensayo de inmunofluorescencia indirecta. MSP1: Proteína de superficie de merozoíto. RESA: del inglés, ring-infected erythrocyte surface antigen, CSP:Proteína de circumsporozoito.

antimalárica más eficaz de todas las evaluadas, quedó claro que SPf66 no alcanzaría un nivel de protección total, por lo que la FIDIC decidió trabajar en el desarrollo de una segunda generación de vacuna multiepítope, multiestadio, basada en subunidades mínimas denominada COLFAVAC.

\section{COLFAVAC (Colombian falciparum vaccine)}

A partir de los obtenidos con SPf66, la FIDIC ha trabajado en el desarrollo de una vacuna de segunda generación, multiepítope, multiestadio, basada en subunidades mínimas, siguiendo la estrategia de síntesis de péptidos en fase sólida. En los múltiples ensayos llevados a cabo se pudo seleccionar este candidato a vacuna, el cual se basa en la selección de péptidos obtenidos a partir de secuencias de proteínas involucradas en la invasión del parásito a la célula diana, los cuales se unían a glóbulos rojos humanos o a células hepáticas HepG2. Estos péptidos son denominados HABPs (del inglés, High Activity Binding Peptides) y se caracterizaron por no mostrar variabilidad genética entre las cepas de parásito; son llamados cHABPs (Conserved HABPs). Sin embargo, se encontró que estos péptidos no fueron reconocidos por el sistema inmune, por lo cual fueron modificados para hacerlos visibles a la respuesta inmune del hospedero mediante la modificación de los residuos críticos en la unión, por aminoácidos con diferente polaridad, pero igual volumen y masa, denominados mHABPs (del inglés Modified High Activity Binding Peptides). La mezcla de diferentes mHABPs provenientes de proteínas del Spz y del mrz, hacen parte de la segunda generación de vacuna contra la malaria frente a $P$. falciparum, desarrollada en la FIDIC que actualmente se encuentra en ensayos preclínicos $(7,65)$. 


\section{CONCLUSIÓN}

Durante varios años de investigación, solo la vacuna RTS,S / AS01 ha alcanzado ensayos clínicos fase III (48), otros candidatos a vacuna continúan siento desarrollados, algunos se encuentran en fases preclínicas y otros han avanzado a fases clínicas; sin embargo, la compleja biología del parásito causante de malaria, la propagación de cepas de $P$. falciparum, los mecanismos de evasión del parásito a la respuesta inmune y su preferencia por el tejido humano y el primate no humano, hacen que las dificultades para la realización de evaluaciones preclínicas sigan siendo un motivo de fracasos durante los estudios clínicos en humanos, por lo cual nuevos modelos animales como ratones quiméricos que admiten el desarrollo completo de las etapas hepáticas y de sangre de P. falciparum son actualmente utilizados (66-68).

Por otra parte, a pesar de los diferentes estudios realizados, la mayoría de los candidatos a vacuna contra la malaria causada por P. falciparum solo han reportado resultados prometedores en retos con cepas homólogas, pero al realizar el desafío con cepas heterólogas, la eficacia llega a ser menor del $10 \%$. Por lo anterior, siguen en curso diversos estudios de candidatos a vacuna que sean capaces de inducir una respuesta inmune protectiva y duradera, para lo cual es necesario tener en cuenta la expresión génica y la alta variabilidad genética del parásito (69-71), así como la diversidad genética del hospedero dada por la inmensa cantidad de polimorfismos de cadena $\beta$ del Complejo Mayor de Histocompatibilidad Clase II (MHC clase II), que es importante para la presentación de péptidos antigénicos al receptor de células T (TCR), permitiendo la inducción de respuestas celulares robustas y de memoria a largo plazo, proceso crítico para la eficacia sostenida de la vacuna (72-74).

\section{CONFLICTOS DE INTERÉS}

Los autores declaran que no existen conflictos de intereses.

\section{FINANCIACIÓN}

Los autores declaran financiación de la Universidad de Boyacá de los medios necesarios para llevar a cabo este artículo de revisión.

\section{AGRADECIMIENTOS}

Los autores queremos agradecer a la Universidad de Boyacá por su apoyo en la trayectoria académica y laboral.

\section{REFERENCIAS}

1. Draper SJ, Sack BK, King $C R$, Nielsen $C M$, Rayner JC, Higgins MK, et al. Malaria Vaccines: Recent Advances and New Horizons. Cell 
Anny Jineth Camargo-Mancipe, Diana Díaz-Arévalo, David Ricardo Salamanca- Jiménez,

Laura Esperanza Cuy-Chaparro, Diego Fernando Camargo-Mancipe

Host Microbe. 2018;24:43-56. https://doi.org/10.1016/j.chom.2018.06.008

2. Miller LH, Ackerman HC, SuX, Wellems TE. Malaria biology and disease pathogenesis: insights for new treatments. Nat Med. 2013;19:156-67. https://doi.org/10.1038/nm.3073

3. Howes RE, Battle KE, Mendis KN, Smith DL, Cibulskis RE, Baird JK, et al. Global Epidemiology of Plasmodium vivax. Am J Trop Med Hyg. 2016;95(6 Suppl):15-34. https://doi. org/10.4269/ajtmh.16-0141

4. Phillips MA, Burrows JN, Manyando C, van Huijsduijnen RH, Van Voorhis WC, Wells TNC. Malaria. Nat Rev Dis Primer. 2017;3:23. https:// doi.org/10.1038/nrdp.2017.50

5. Cowman AF, Tonkin CJ, Tham W-H, Duraisingh MT. The Molecular Basis of Erythrocyte Invasion by Malaria Parasites. Cell Host Microbe. 2017;22(2):232-245. https://doi.org/10.1016/j.chom.2017.07.003

6. Patarroyo ME, Alba MP, Rojas-Luna R, Bermúdez A, Aza-Conde J. Functionally relevant proteins in Plasmodium falciparum host cell invasion. Immunotherapy. 2017;9:131-55. https:// doi.org/10.2217/imt-2016-0091
7. Patarroyo ME, Bermúdez A, Patarroyo MA. Structural and Immunological Principles Leading to Chemically Synthesized, Multiantigenic, Multistage, Minimal Subunit-Based Vaccine Development. Chem Rev. 2011;111(5):3459-507. https://doi.org/10.1021/cr100223m

8. World Health Organization. World malaria report 2018. 2018; 1-210 $p$.

9. World Health Organization, World Health Organization, Global Malaria Programme. Global technical strategy for malaria, 2016-2030. World Health Organization; 2015. 1-35 p.

10. Goh YS. Mcguire D, Renia L.Vaccination With Sporozoites: Models and Correlates of Protection. Front Immunol. 2019;10:18. https://doi. org/10.3389/fimmu.2019.01227

11. De SL, Stanisic DI, van Breda K, Bellete B, Harris I, McCallum F, et al. Persistence and immunogenicity of chemically attenuated blood stage Plasmodium falciparum in Aotus monkeys. Int J Parasitol. 2016;46:581-91. https://doi.org/10.1016/j.ijpara.2016.05.002

12. Herrera $S$, Perlaza BL, Bonelo A, Arévalo-Herrera M. Aotus monkeys: their great value for anti-malaria vaccines and drug testing. Int J Parasitol. 2002;32(13):1625-35. https://doi. org/10.1016/s0020-7519(02)00191-1 
13. Langhorne J, Buffet P, Galinski M, Good M, Harty J, Leroy D, et al. The relevance of non-human primate and rodent malaria models for humans. Malar J. 2011;10:1-23. https://doi. org/10.1186/1475-2875-10-23

14. Nino-Vasquez JJ, Vogel D, Rodríguez R, Moreno A, Patarroyo ME, Pluschke G, et al. Sequence and diversity of DRB genes of Aotus nancymaae, a primate model for human malaria parasites. Immunogenetics. 2000;51(3):21930. https://doi.org/10.1007/s002510050035

15. Suárez M. CF, Patarroyo MA, Patarroyo ME. Characterisation and comparative analysis of MHC-DPA1 exon 2 in the owl monkey (Aotus nancymaae). Gene.2011;470(1-2):37-45. https://doi.org/10.1016/j.gene.2010.09.006

16. Cardenas PP, Suárez CF, Martínez P, Patarroyo $\mathrm{ME}$, Patarroyo MA. MHC class I genes in the owl monkey: mosaic organisation, convergence and loci diversity. Immunogenetics. 2005;56(11):818-32. https://doi.org/10.1007/ s00251-004-0751-5

17. Baquero JE, Miranda S, Murillo O, Mateus H, Trujillo E, Suárez C, et al. Reference strand conformational analysis (RSCA) is a valuable tool in identifying MHC-DRB sequences in three species of Aotus monkeys. Immunogenetics. 2006;58(7):590-7. https://doi.org/10.1007/s00251-006-0101-x
18. Guerrero JE, Pacheco DP, Suárez CF, Martínez P, Aristizabal F, Moncada CA, et al. Characterizing T-cell receptor gamma-variable gene in Aotus nancymaae owl monkey peripheral blood. Tissue Antigens.2003;62(6):472-82. https:// doi.org/10.1046/j.1399-0039.2003.00130.x

19. Jones TR, Obaldia N, Hoffman SL, Gramzinski RA. Repeated infection of Aotus monkeys with Plasmodium falciparum induces protection against subsequent challenge with homologous and heterologous strains of parasite. Am J Trop Med Hyg. 2000;62(6):675-80. https:// doi.org/10.4269/ajtmh.2000.62.675

20. Suárez CF, Pabón L, Barrera A, Aza-Conde J, Patarroyo MA, Patarroyo ME. Structural analysis of owl monkey MHC-DR shows that fully-protective malaria vaccine components can be readily used in humans. Biochem Biophys Res Commun. 2017;491(4):1062-9. https:// doi.org/10.1016/j.bbrc.2017.08.012

21. Minkah NK, Schafer C, Kappe SHI. Humanized Mouse Models for the Study of Human Malaria Parasite Biology, Pathogenesis, and Immunity. Front Immunol. 2018;1-9. https:// doi.org/10.3389/fimmu.2018.00807

22. Nussenzweig RS, Vanderberg J, Most H, Orton C. Protective Immunity produced by the Injection of X-irradiated Sporozoites of Plasmodium 
Anny Jineth Camargo-Mancipe, Diana Díaz-Arévalo, David Ricardo Salamanca- Jiménez,

Laura Esperanza Cuy-Chaparro, Diego Fernando Camargo-Mancipe

berghei. Nature. 1967;216:160-2. https://doi. org/10.1038/216160a0

23. Nussenzweig RS, Vanderberg JP, Most $\mathrm{H}$, Orton C. Specificity of Protective Immunity produced by X-irradiated Plasmodium berghei Sporozoites. Nature.1969;222(5192):488-9. https://doi.org/10.1038/222488a0

24. Vanderberg JP, Nussenzweig RS, Most $H$, Orton CG. Protective immunity produced by the injection of $x$-irradiated sporozoites of Plasmodium berghei. II. Effects of radiation on sporozoites. J Parasitol. 1968;54(6):117580. https://doi.org/10.2307/3276987

25. Nussenzweig RS, Zavala F. A Malaria Vaccine Based on a Sporozoite Antigen.NEngl J Med.1997;336(2):128-30. https://doi. org/10.1056/NEJM199701093360210

26. Nussenzweig V, Nussenzweig RS. Rationale for the Development of an Engineered Sporozoite Malaria Vaccine. Adv Immunol.1989;45:283-334. https://doi.org/10.1016/s0065-2776(08)60695-1

27. Epstein JE, Tewari K, Lyke KE, Sim BKL, BiIlingsley PF, Laurens MB, et al. Live Attenuated Malaria Vaccine Designed to Protect Through Hepatic CD8+ T Cell Immunity. Science. 2011;334(6055):475-80. https://doi. org/10.1126/science. 1211548
28. Hill AV, Biswas $S$, Draper $S$, Rampling $T$, Reyes-Sandoval A. Towards a multi-antigen multi-stage malaria vaccine. Malar J. 2014;13(S1):031. . https://doi.org/10.1186/1475-2875-13-S1-031

29. Tolia NH, Enemark EJ, Sim BKL, Joshua-Tor L. Structural Basis for the EBA-175 Erythrocyte Invasion Pathway of the Malaria Parasite Plasmodium falciparum. Cell. 2005;122(2):18393. https://doi.org/10.1016/j.cell.2005.05.033

30. Sim BKL, Chitnis CE, Wasniowska K, Millert $\mathrm{LH}$. Receptor and ligand domains for invasion of erythrocytes by Plasmodium falciparum. 1994;264:1941-3. https://doi.org/10.1126/ science. 8009226

31. Jones TR, Narum DL, Gozalo AS, Aguiar J, Fuhrmann SR, Liang $\mathrm{H}$, et al. Protection of Aotus Monkeys by Plasmodium falciparum EBA-175 Region II DNA Prime-Protein Boost Immunization Regimen. J Infect Dis. 2001;183(2):30312. https://doi.org/10.1086/317933

32. Volz JC, Yap A, Sisquella X, Thompson JK, Lim NTY, Whitehead LW, et al. Essential Role of the PfRh5/PfRipr/CyRPA Complex during Plasmodium falciparum Invasion of Erythrocytes. Cell Host Microbe. 2016;20:60-71. https://doi. org/10.1016/j.chom.2016.06.004 
33. Tran TM, Ongoiba A, Coursen J, Crosnier $C$, Diouf $A$, Huang $C-Y$, et al. Naturally Acquired Antibodies Specific for Plasmodium falciparum Reticulocyte-Binding Protein Homologue 5 Inhibit Parasite Growth and Predict Protection From Malaria. J Infect Dis. 2014;209:78998. https://doi.org/10.1093/infdis/jit553

34. Patel SD, Ahouidi AD, Bei AK, Dieye TN, Mboup S, Harrison SC, et al. Plasmodium falciparum Merozoite Surface Antigen, PfRH5, Elicits Detectable Levels of Invasion-Inhibiting Antibodies in Humans. J Infect Dis. 2013;208:167987. https://doi.org/10.1093/infdis/jit385

35. Chen L, Xu Y, Healer J, Thompson JK, Smith BJ, Lawrence MC, et al. Crystal structure of PfRh5, an essential P. falciparum ligand for invasion of human erythrocytes. eLife. 2014;3:1-10. https://doi.org/10.7554/eLife.04187.001

36. Sheehy SH, Duncan CJ, Elias SC, Choudhary P, Biswas S, Halstead FD, et al. ChAd63-MVAvectored Blood-stage Malaria Vaccines Targeting MSP1 and AMA1: Assessment of Efficacy Against Mosquito Bite Challenge in Humans. Mol Ther. 2012;20(12):2355-68. https://doi. org/10.1038/mt.2012.223

37. Draper SJ, Moore AC, Goodman AL, Long CA, Holder AA, Gilbert SC, et al. Effective induction of high-titer antibodies by viral vector vaccines. Nat Med. 2008;14:819-21. https:// doi.org/10.1038/nm.1850

38. de Cassan SC, Forbes EK, Douglas AD, Milicic A, Singh B, Gupta P, et al. The Requirement for Potent Adjuvants To Enhance the Immunogenicity and Protective Efficacy of Protein Vaccines Can Be Overcome by Prior Immunization with a Recombinant Adenovirus. J Immunol. 2011;187:2602-16. https://doi.org/10.4049/ jimmunol.1101004

39. Douglas AD, Baldeviano GC, Lucas CM, Lugo-Roman LA, Crosnier C, Bartholdson SJ, et al. A PfRH5-Based Vaccine Is Efficacious against Heterologous Strain Blood-Stage Plasmodium falciparum Infection in Aotus Monkeys. Cell Host Microbe. 2015;17:130-9. https://doi.or$\mathrm{g} / 10.1016 / \mathrm{j}$.chom.2014.11.017

40. Srinivasan P, Beatty WL, Diouf A, Herrera R, AmbroggioX, Moch JK, etal. Binding of Plasmodium merozoite proteins RON2 and AMA1 triggers commitment to invasion. Proc Natl Acad Sci. 2011;108:13275-80. https://doi.org/10.1073/pnas.1110303108

41. Tyler JS, Boothroyd JC. The C-Terminus of Toxoplasma RON2 Provides the Crucial Link between AMA1 and the Host-Associated Invasion Complex. Soldati-Favre D, editor. PLoS Pathog. 2011;7:1-12. https://doi.org/10.1371/ journal.ppat.1001282 
42. Mital J, Meissner M, Soldati D, Ward GE. Conditional Expression of Toxoplasma gondii Apical Membrane Antigen-1 (TgAMA1) Demonstrates That TgAMA1 Plays a Critical Role in Host Cell Invasion. Mol Biol Cell. 2005;16(9):43419. https://doi.org/10.1091/mbc.e05-04-0281

43. Payne RO, Milne KH, Elias SC, Edwards NJ, Douglas $A D$, Brown RE, et al. Demonstration of the Blood-Stage Plasmodium falciparum Controlled Human Malaria Infection Model to Assess Efficacy of the P. falciparum Apical Membrane Antigen 1 Vaccine, FMP2.1/AS01. J Infect Dis. 2016;213:1743-51. https://doi. org/10.1093/infdis/jiw039

44. Spring MD, Cummings JF, Ockenhouse CF, Dutta S, Reidler R, Angov E, et al. Phase 1/2a Study of the Malaria Vaccine Candidate Apical Membrane Antigen-1 (AMA-1) Administered in Adjuvant System AS01B or AS02A. Beeson JG, editor. PLoS ONE. 2009;4(4):1-13. https:// doi.org/10.1371/journal.pone.0005254

45. Srinivasan $P$, Baldeviano GC, Miura K, Diouf A, Ventocilla JA, Leiva KP, et al. A malaria vaccine protects Aotus monkeys against virulent Plasmodium falciparum infection. Npj Vaccines. 2017;2(1):1-10. https://doi.org/10.1038/ s41541-017-0015-7
46. Burns JM, Miura K, Sullivan J, Long CA, Barnwell JW. Immunogenicity of a chimeric Plasmodium falciparum merozoite surface protein vaccine in Aotus monkeys. Malar J. 2016;1-7. https://doi.org/10.1186/s12936-016-1226-5

47. Bejon P, Lusingu J, Olotu A, Leach A, Lievens $M$, Vekemans J, et al. Efficacy of RTS,S/AS01E Vaccine against Malaria in Children 5 to 17 Months of Age. N Engl J Med. 2008;359:252132. https://doi.org/10.1056/NEJMoa0807381

48. Brian Greenwood. Efficacy and safety of RTS,S/AS01 malaria vaccine with or without a booster dose in infants and children in Africa: final results of a phase 3 , individually randomised, controlled trial. The Lancet. 2015;386:60721-8. https://doi.org/10.1016/ S0140-6736(15)60721-8

49. Vekemans J, Leach A, Cohen J. Development of the RTS,S/AS malaria candidate vaccine. Vaccine. 2009;27:67-71. https://doi.org/10.1016/j.vaccine.2009.10.013

50. Cohen J, Nussenzweig V, Vekemans J, Leach A. From the circumsporozoite protein to the RTS,S/ AS candidate vaccine. Hum Vaccin. 2010;6:906. https://doi.org/10.4161/hv.6.1.9677

51. Pichyangkul S, Tongtawe $P$, Kum-Arb U, Yongvanitchit $K$, Gettayacamin M, Hollingdale MR, 
et al. Evaluation of the safety and immunogenicity of Plasmodium falciparum apical membrane antigen 1, merozoite surface protein 1 or RTS,S vaccines with adjuvant system AS02A administered alone or concurrently in rhesus monkeys. Vaccine. 2009;28(2):452-62. https:// doi.org/10.1016/j.vaccine.2009.10.022

52. Kester KE, Stewart AV, Walsh DS, Voss G, Tongtawe $P$, Ballou WR, et al. Safety and immunogenicity of RTS,S + TRAP malaria vaccine, formulated in the AS02A adjuvant system, in infant Rhesus monkeys. Am J Trop Med Hyg. 2004;70:499-509. https://doi.org/10.4269/ ajtmh.2004.70.499

53. Kester KE, Cummings JF, Ofori-Anyinam $\mathrm{O}$, Ockenhouse CF, Krzych U, Moris P, et al. Randomized, Double-Blind, Phase 2a Trial of Falciparum Malaria Vaccines RTS,S/AS01B and RTS,S/AS02A in Malaria-Naive Adults: Safety, Efficacy, and Immunologic Associates of Protection. J Infect Dis. 2009;200:337-46. https:// doi.org/10.1086/600120

54. Garçon N, Chomez P, Van Mechelen M. GlaxoSmithKline Adjuvant Systems in vaccines: concepts, achievements and perspectives. Expert Rev Vaccines. 2007;6:723-39. https:// doi.org/10.1586/14760584.6.5.723
55. Walsh D, Gettayacamin M, Leitner W, Lyon J, Stewart V, Marit G, et al. Heterologous prime-boost immunization in rhesus macaques by two, optimally spaced particle-mediated epidermal deliveries of Plasmodium falciparum circumsporozoite protein-encoding DNA, followed by intramuscular RTS,S/AS02A. Vaccine. 2006;24:4167-78. https://doi.org/10.1016/j.vaccine.2006.02.041

56. Stewart VA, McGrath SM, Dubois PM, Pau MG, Mettens $P$, Shott J, et al. Priming with an Adenovirus 35-Circumsporozoite Protein (CS) Vaccine followed by RTS,S/AS01B Boosting Significantly Improves Immunogenicity to Plasmodium falciparum CS Compared to That with Either Malaria Vaccine Alone. Infect Immun. 2007;75(5):228390. https://doi.org/10.1128/IAI.01879-06

57. Dunachie SJ, Walther M, Vuola JM, Webster DP, Keating SM, Berthoud T, et al. A clinical trial of prime-boost immunisation with the candidate malaria vaccines RTS,S/AS02A and MVA-CS. Vaccine. 2006;(15):2850-9. https:// doi: 10.1016/j.vaccine.2005.12.041

58. GwADz RW, Cochrane H, Nussenzweig V, Nussenzweig RS. Preliminary studies on vaccination of rhesus monkeys with irradiated sporozoites of Plasmodium knowlesi and characterization of surface antigens of these parasites. Bull World Health Organ. 1979;165-73. 
59. Hoffman SL, Goh LML, Luke TC, Schneider I, Le TP, Doolan DL, et al. Protection of Humans against Malaria by Immunization with Radiation-Attenuated Plasmodium falciparum Sporozoites. J Infect Dis. 2002;185:1155-64. https://doi.org/10.1086/339409

60. Patarroyo ME, Romero P, Torres ML, Clavijo $P$, Moreno A, Martínez A et al. Induction of protective immunity against experimental infection with malaria using synthetic peptides. Nature. 1987;328:629-32. https://doi.or$\mathrm{g} / 10.1038 / 328629 \mathrm{a} 0$

61. Noya G. O, Berti YG, Noya BA d., Borges R, Zerpa N, Urb ez JD, et al. A Population-Based Clinical Trial with the SPf66 Synthetic Plasmodium falciparum Malaria Vaccine in Venezuela. J Infect Dis. 1994;170:396-402. https://doi. org/10.1093/infdis/170.2.396

62. Sempertegui F, Estrella B, Moscoso J, Piedrahita L, Hernández D, Gaybor J et al. Safety, immunogenicity and protective effect of the SPf66 malaria synthetic vaccine against Plasmodiumfalciparum infection in a randomized double-blind placebo-controlled field trial fin an endemic area of Ecuador. Vaccine. 1994;337-42. https://doi.org/10.1016/0264-410X(94)90098-1

63. Valero M, Amador R, Aponte J, Narvaez A, Galindo $C$, Silva $Y$ et al. Evaluation of SPf66 malaria vaccine during a 22-month follow-up field trial in the Pacific coast of Colombia. Vaccine. 1996;14:1466-70. https://doi. org/10.1016/S0264-410X(96)00070-9

64. Valero MV, Amador LR, Galindo C, Figueroa J, Bello MS, Murillo LA, et al. Vaccination with SPf66, a chemically synthesised vaccine, against Plasmodium falciparum malaria in Colombia. The Lancet. 1993;341:705-10. https:// doi.org/10.1016/0140-6736(93)90483-W

65. Patarroyo ME, Patarroyo MA. Emerging Rules for Subunit-Based, Multiantigenic, Multistage Chemically Synthesized Vaccines. Acc Chem Res. 2008;41:37786. https://doi.org/10.1021/ar700120t

66. Vaughan AM, Mikolajczak SA, Wilson EM, Grompe M, Kaushansky A, Camargo N, et al. Complete Plasmodium falciparum liver-stage development in liver-chimeric mice. J Clin Invest. 2012;122:361828. https://doi.org/10.1172/JCl62684

67. Mikolajczak SA, Vaughan AM, Kangwanrangsan $N$, Roobsoong $W$, Fishbaugher $M$, Yimamnuaychok $N$, et al. Plasmodium vivax Liver Stage Development and Hypnozoite Persistence in Human Liver-Chimeric Mice. Cell Host Microbe. 2015;17(4):526-35. https://doi. org/10.1016/j.chom.2015.02.011 
68. Sack BK, Mikolajczak SA, Fishbaugher M, Vaughan AM, Flannery EL, Nguyen T, et al. Humoral protection against mosquito bite-transmitted Plasmodium falciparum infection in humanized mice. Npj Vaccines. 2017;2(1):1-11. https://doi.org/10.1038/s41541-017-0028-2

69. Weiss GE, Crabb BS, Gilson PR. Overlaying Molecular and Temporal Aspects of Malaria Parasite Invasion. Trends Parasitol. 2016;32(4):28495. https://doi.org/10.1016/j.pt.2015.12.007

70. Weiss GE, Gilson PR, Taechalertpaisarn T, Tham W-H, de Jong NWM, Harvey KL, et al. Revealing the Sequence and Resulting Cellular Morphology of Receptor-Ligand Interactions during Plasmodium falciparum Invasion of Erythrocytes. Blackman MJ, editor. PLOS Pathog. 2015;11(2):e1004670. https://doi.org/10.1371/journal.ppat.1004670

71. Moser KA, Drábek EF, Dwivedi A, Crabtree J, Stucke EM, Dara A, et al. Strains used in whole organism Plasmodium falciparum vaccine trials differ in genome structure, sequence, and immunogenic potential. Genomics; 2019 https://doi.org/10.1101/684175

72. Lyke KE, Fernández-Viňa MA, Cao K, Hollenbach J, CoulibalyD, KoneAK, etal. Association of HLAalleles with Plasmodium falciparum severity in Malian children. Tissue Antigens. 2011;77(6):562-71. https:// doi.org/10.1111/j.1399-0039.2011.01661.x
73. Matern BM, Olieslagers TI, Voorter CEM, Groeneweg M, Tilanus MGJ. Insights into the polymorphism in HLA-DRA and its evolutionary relationship with HLA Haplotypes. HLA. 2019;95(2):13730. https://doi.org/10.1111/tan.13730

74. La Gruta NL, Gras S, Daley SR, Thomas PG, Rossjohn J. Understanding the drivers of $\mathrm{MHC}$ restriction of T cell receptors. Nat Rev Immunol.2018;18(7):46778. https://doi.org/10.1038/ s41577-018-0007-5 